\section{STOA NEWS}

\section{Integrated E-ticketing for Public Transport and Touristic Sites}

The final report of the STOA project on Integrated E-ticketing was approved by the STOA panel in October. The results of the project were presented by Maike Puhe, ITAS, at a STOA panel meeting in Strasbourg on 10 October and at a STOA workshop held at the Brussels premises of the European Parliament on 16 October. For the workshop visit: http://www.europarl.europa.eu/ stoa/cms/home/events/workshops/eticketing.

The project deals with the development of integrated ticketing systems for public transport and touristic sites in cities. The overall idea of integrated ticketing is to combine several modes of transport (e.g. tram, bus, car sharing, and bike sharing) and other interesting fields of application (e.g. leisure offers and tourist attractions) on a single ticket. That way, integrated ticketing schemes aim at facilitating the combination of transport modes and the transfer for its users. Ticket issuers hope to receive a deeper insight into their customers' habits and preferences. Good accessibility and information are also key determinants of the attractiveness of touristic and leisure sites. Modern multi-application smart cards are able to incorporate different fields of application (e.g. leisure and touristic schemes) and the most successful schemes in Asia are already using such special features. While technologies are already available and ready to meet multifunctional requirements, e-ticketing has not yet been implemented on a transnational scale in Europe. A prerequisite for the implementation is that a number of stakeholders, such as public transport operators and authorities, financial service providers, telecommunications operators, and the tourism industry, are working together to combine their products on a single card. One of the main barriers for the functioning are not tech- nological aspects but the difficulties in agreeing on the same standards. Obviously, there is a need for organizational, management, and governmental changes to realize such systems on a wider scale. The final report provides an overview on the technical details of integrated e-ticketing and takes a closer look at each stakeholder's role and potential benefit in the e-ticketing domain. A particular focus lies on potential and existing endusers. The report comprises a review of existing systems of urban transport ticketing assessing their level of integration into touristic sites and into urban transport systems. Currently the results of the discussions at the STOA workshop are being integrated into the final policy options chapter of the report. The full report will soon be available for download at STOA's webpage: http://www.europarl.europa.eu/stoa/cms/home/ publications/studies.

\section{Contact}

Maike Puhe, Email: maike.puhe@kit.edu

\section{$\langle\gg$}

\section{Cloud Computing}

Cloud computing is one of the most controversially discussed developments in recent years. The opportunities using powerful computing resources on demand via the web are considered as a possible driver for the growth of the European economy. Especially cost savings as well as increased productivity and mobility are seen as key elements by many experts. However, there are also critics arguing that cloud computing bears too many economic, social, and technical risks or even dismissing the potentials as a whole. The STOA project on cloud computing, coordinated by Fraunhofer ISI on behalf of ETAG, deals with a broad spectrum of issues connected with cloud computing, some of them were discussed at a workshop at the European Parliament in October. A special sub-report on Social Network Sites was prepared by colleagues from the Institute of Technology Assessment of the Austrian Academy 
of Sciences (ITA) and was recently delivered to the Parliament. The integrated final report of the project will be available in January 2014.

\section{Workshop on Cloud Computing at the European Parliament}

The interim results on the socio-economic potentials of cloud computing were presented on behalf of ETAG by Arnd Weber (ITAS) at a workshop held at the European Parliament on 2 October. The workshop aimed at discussing the potentials of cloud computing in Europe, in particular the challenges and problems that need to be addressed to foster the possible potentials. Several experts gave insight into challenges from different perspectives. Caspar Bowden, an independent privacy researcher, commented critically on the EU data protection strategy for the cloud, which was presented by Jorge Gasos from the European Commission's DG Connect. The business perspective on the economics of the cloud computing market was presented by Theo Lynn from the Irish Center for Cloud Computing and Commerce and the issue of the future competitiveness of Europe's ICT sector was addressed by Reinhilde Veugelers from the University of Leuven. The consumer perspective on options and privacy risks of cloud computing was represented by Chiara Giovannini from ANEC, a European consumer organization for technical standardization issues.

\section{Contact}

Timo Leimbach,

Email: timo.leimbach@isi.fraunhofer.de Dr. Arnd Weber, Email: arnd.weber@kit.edu

\section{Report on Social Network Sites}

Cloud computing and social network sites (SNS) are part of the same societal transformation prominently exemplifying a paradigm shift stating that "the network is the computer". While cloud computing includes a variety of technical concepts, SNS represent a broad scope of services and applications mostly addressing end-users. Interre- lations are particularly given as regards privacy and security challenges. The report is structured in four main parts: The first part (sections 1-3) gives a broad overview on the evolution of SNS describing the main stages in the development process, the major factors determining the current state-of-the-art including insights into user motivations. Section 3 presents the main driving factors determining the current SNS, their main characteristics as well as the core functionality of SNS. Based on these elaborations the second part (section 4) gives an overview on the spectrum of identified societal impacts, discusses the role of SNS as a form of a (digital) semi-public space, as well as their potential for political participation and knowledge production. The third part (section 5) analyses in more depth the privacy implications of SNS including some technical aspects and privacy-by-design concepts. The final part (section 6) draws conclusions referring to possible options to address the challenges identified.

As regards privacy issues connected with SNS, the report highlights the lacking distinction between user information, interactions, and content as the crux of SNS. The combination of these aspects enables SNS to gain deep and farreaching insights into user behavior and identity. Recent innovations such as the social graph (grounding on mathematical graph theory) aim at systematically mapping the variety of different relations and interactions and thus aggravate these problematic aspects. These developments multiply the existing barriers for users to exercise their right of informational self-determination. The limited options for individuals to protect their privacy complicate further if the SNS architectures do not include appropriate and effective protection mechanisms. This underlines the demand for privacy-by-design concepts as integral parts of SNS environments. Respective strategies need to deal with at least two core problems of contemporary privacy protection: a disclosure-by-default paradigm exemplified by SNS, i.e. the widespread availability of personal information as standard mode; and the related increase in personal identifiable information reinforced by a convergence of personal and non-personal data as one result of the multiple interactions, not least between personal and 
non-personal entities. Contemporary SNS affect several different types of privacy (such as communication, data and image, behavior and action, location). Considering emerging trends related to SNS, privacy impacts might increase further with social plugins and graphs, biometrics and face recognition technologies, as well as mobile SNS usage and location-based services as fast growing markets. Addressing these privacy challenges demands not least a shift of the prevailing disclosure-by-default paradigm towards a setting where privacy-by-design and privacy-by-default are the leading principles. Measures to stimulate this shift might be:

- Enforce content encryption as standard

- Foster anonymity and pseudonymity

- Strengthen freedom of information and transparency

- Raise awareness for privacy and transparency

- Stimulate innovation for privacy by design

- Strengthen the role of Data Protection Authorities to improve checks and balances

These measures are particularly salient in the face of the recent scandals revealing large-scale surveillance of individuals on a global level. While the collateral damage caused by these scandals is yet unpredictable, they highlight urgency for a revitalization of privacy - a concept that is strongly connected to the need to recover the individuals' trust in the system.

The full report will soon be available at STOA's webpage: http://www.europarl.europa. $\mathrm{eu} / \mathrm{stoa} / \mathrm{cms} /$ home/publications/studies.

\section{Contact}

Stefan Strauß, Email: sstrauss@oeaw.ac.at Dr. Michael Nentwich, Email: mnent@oeaw.ac.at

\section{$\langle\gg$}

\section{"Cutting Food Waste" and "Plant Breeding and Innovative Agriculture"}

\begin{abstract}
ITAS was responsible for two studies carried out within the framework of the STOA project "Technology options for feeding 10 billion people". The final reports of both were presented by the authors at a STOA panel meeting on 24 October and at a meeting of the European Parliament's standing committee on Agriculture and Rural Development (AGRI) on 5 November in Brussels. Both studies are available at STOA's webpage: http://www.europarl.europa.eu/stoa/cms/home/ publications/studies.
\end{abstract}

\section{Cutting Food Waste}

The report highlights the range of possible definitions of the terms "food loss" and "food waste", provides an overview of current research activities and political initiatives, depicts the reasons of the arising of losses along the food chain and deals with adverse environmental and economic impacts of food wastage. The report provides insights into the existing data situation and offers the results of own calculations based on the methodology of SIK and FAO's "food balance sheets". Recent studies, including own model calculations presented in the report, consistently come to the conclusion that one of the largest savings potential in Europe is at household level. Therefore food waste generation on household level is considered in more detail. The report looks into avoidable and unavoidable shares of household food waste and its composition, dietary patterns across EU-27 as well as impacts of the economic situation on household consumption. Furthermore, the results of a survey on food waste generation in households, carried out jointly at the Joint Research Centre in Ispra, the University of Bologna and the Karlsruhe Institute of Technology, are presented. The study gives an overview of measures and instruments for food waste reduction under discussion, taking into account the experiences already gained in different countries. The focus is on instruments 
that are considered in literature or in the current debate as particularly useful, easy to implement and able to achieve long-term gains and/or that have already proven their effectiveness in practice. In the concluding part the report identifies hotspots of food wastage within EU-27 and gives an overview of the measures and instruments under discussion to reduce food waste, following the stages of the food supply chain. Based on the discussion of possible approaches to reduce food waste, a broad scope of options for action on the European and national level are presented, such as - among others an amendment of European marketing standards based not on the external appearance of a food product but on its quality for human consumption.

\section{Contact}

Carmen Priefer, Email: carmen.priefer@kit.edu Juliane Jörissen, Email: juliane.joerissen@kit.edu Rainer Bräutigam

Email: klaus-rainer.bräutigam@kit.edu

\section{Plant Breeding and Innovative Agriculture}

The objective of the study is to analyze how farming management concepts, practices and technologies, including plant breeding technologies, could enable sustainable intensification of crop production, with the aim to increase food production and support food security. The scope of the study encompasses agriculture in developing countries, in industrialized countries (Europe), small-scale and large-scale farming, extensive and intensive agricultural production systems, and low and high tech production practices. The assessment is restricted to crop production. The main topics are: Reducing the yield gap - sustainable intensification and improving crop management; increasing the yield potential - plant breeding, and reducing crop losses - improving harvest and postharvest procedures.

With respect to sustainable intensification for reducing the yield gap, the report identifies the need for a stronger focus on maintenance and enhancement of soil fertility and on exploitation of agro-ecological mechanism in order to stabilize achieved high yield levels in favorable areas, to realize more of existing yield potentials, and to increase the resilience of farming systems. Efforts should be undertaken to explore combinations and mutual benefits between input use efficiency and soil fertility improvement approaches (e.g., precision agriculture and conservation agriculture). As regards plant breeding, the report sees a need for research on marker-assisted selection and SMART breeding as very promising breeding technologies. Hybrid breeding research is regarded to remain of high importance. Progress in organic breeding is needed so that organic farming can take part in an overall increase of yield potentials. Modern breeding technologies should be assessed with regard to their compatibility with the principles of organic farming. Participatory plant breeding as developed to better serve the needs of small-scale farmers in developing countries could be an approach to address European semi-subsistence farming which would need public support. Overall, the report states that modern breeding technologies open new possibilities to create genetic variation and to improve selection, but conventional breeding technologies will remain important. Technologies for reducing harvest and postharvest crop losses are available, the report, however, addresses a number of obstacles to bring them into practice particularly among small poor farmers. Technologies are often not suitable in scale, and they are associated with high investment costs. Most of them require innovations throughout the whole food supply chain.

\section{Contact}

Dr. Rolf Meyer, Email: rolf.meyer@kit.edu 